\title{
Recognition-Induced Transformation of Microspheres into Vesicles Morphology and Size Control
}

Oktay Uzun, Amitav Sanyal, Hiroshi Nakade, Raymond J. Thibault and Vincent M. Rotello*

Department of Chemistry, University of Massachusetts, Amherst, Massachusetts, 01003

\section{Supplementary Material}

\section{Experimental Section}

All chemicals were reagent grade, purchased from Aldrich or Merck, and were used as received. Thymine-1-acetic acid was recrystallized from distilled water prior to use; all other chemicals were used as obtained. All reactions were performed under argon atmosphere. Monomers (styrene and 4-chloromethylstyrene) were purified from the inhibitor by a short alumina plug. ${ }^{1} \mathrm{H}$ NMR spectra were recorded with $3 \mathrm{mg} / \mathrm{mL}$ polymer solutions in $\mathrm{CDCl}_{3}$ on a Bruker $200 \mathrm{Mhz}$ and $400 \mathrm{Mhz}$ spectrometers. Infrared spectra were recorded using either $\mathrm{KBr}$ plates or a solution cell on a MIDAC 2000 FTIR spectrometer. UV-Vis spectra were recorded on a Hewlett Packard 8452A photodiode array spectrophotometer. $\mathrm{CHCl}_{3}$ was spectroscopic grade and distilled from $\mathrm{CaH}_{2}$ prior to use. $\mathrm{CDCl}_{3}$ was obtained from Cambridge Isotopes Laboratories and stored over molecular sieves.

Synthesis. Precursor of polymers 1a, $\mathbf{2 a}$ and $\mathbf{2 b}$ were prepared via AIBN-mediated free radical polymerization of Styrene and Chloromethyl styrene(CMS) (PDI 1.4). Polymer $\mathbf{1 b}$ and 1c were synthesized via nitroxide-mediated free-radical polymerization. ${ }^{1}$ In a typical $100 \%$ functionalization of the CMS sites reaction, the precursor polymer (1.0 CMS equivalent), DAP (1.1 eq.), and $\mathrm{K}_{2} \mathrm{CO}_{3}$ (1.5 eq.) were dissolved in a minimum volume of $\mathrm{N}, \mathrm{N}$-dimethylformamide (DMF) and stirred at $70^{\circ} \mathrm{C}$ under argon atmosphere overnight. Dropwise addition of water resulted in precipitation of the polymer. The white 
solid was filtered, sonicated in methanol, filtered again and dried under vacuum overnight (85-90\% yield).

Polymer 2a and $\mathbf{2 b}$, thymine and $N$-methylated thymine polymer, respectively, prepared by post-functionalization of styrene and 4-(chloromethyl)styrene random monoblock copolymer with appropriate thymine derivative under identical conditions to those used for polymer 1. Conversion was determined using side-chain $\mathrm{CH}_{2}$ group resonance with NMR. When 50\% functionalization of the CMS sites was desired for Polymer 2a and $\mathbf{2 b}$, substituent and base amounts were calculated accordingly, and average conversion ratio was determined with NMR as described.

Characterization. Number-averaged molecular weights $\left(M_{\mathrm{n}}\right)$ and polydispersity indices (PDI) of the polymers in THF solutions were determined from gel permeation chromatograms (GPC) acquired on an in-house built GPC system using PL Caliber data collection software, three-column set (Polymer Labs, Inc.; PLgel 5 mm columns, $300 \mathrm{x}$ $7.5 \mathrm{~mm}, 10^{3}, 10^{4}$, and $10^{5} \AA$ pore size), with an RI (Waters 403 ) detector. The system was calibrated with respect to polystyrene standards (Polymer Labs, Inc.). 
Polymer 1a, 2a and 2b chloromethylstyrene precursor PS/CMS (5k)-NMR
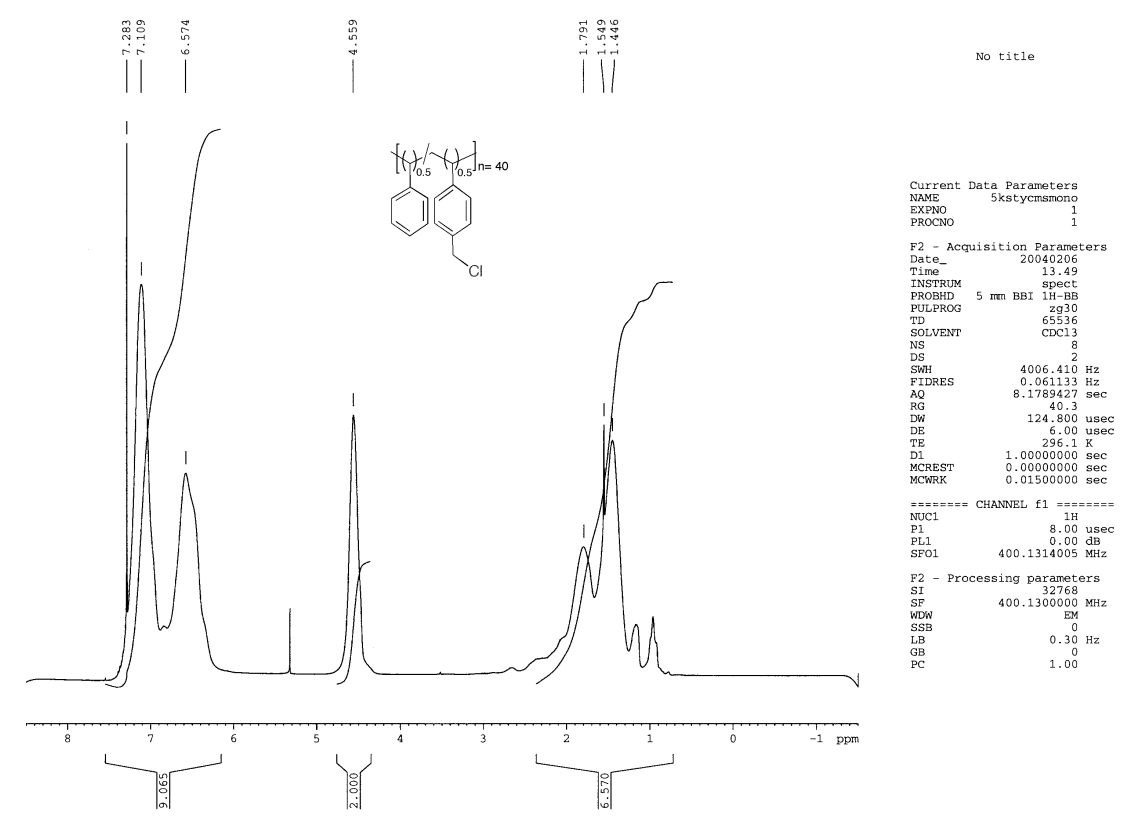

Polymer 1a PS/DAP(5k,100\%)-NMR

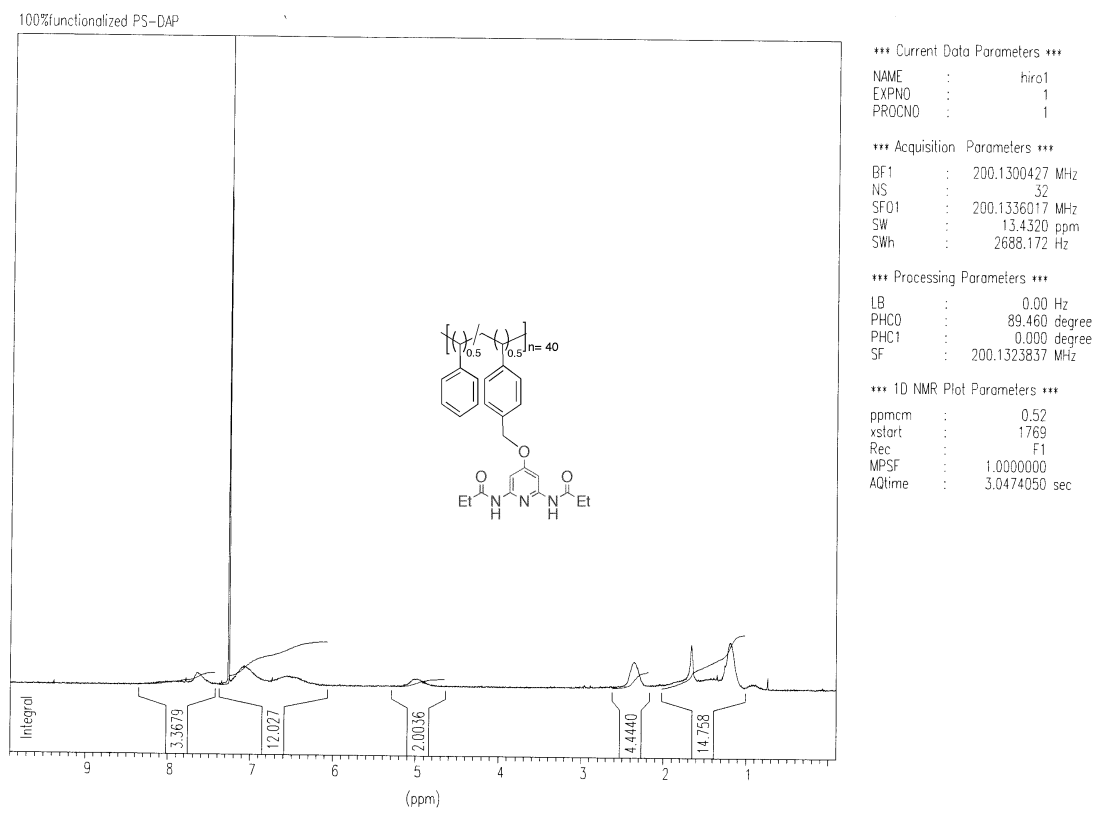




\section{Polymer 2a PS/THY(5k,50\%)-NMR}

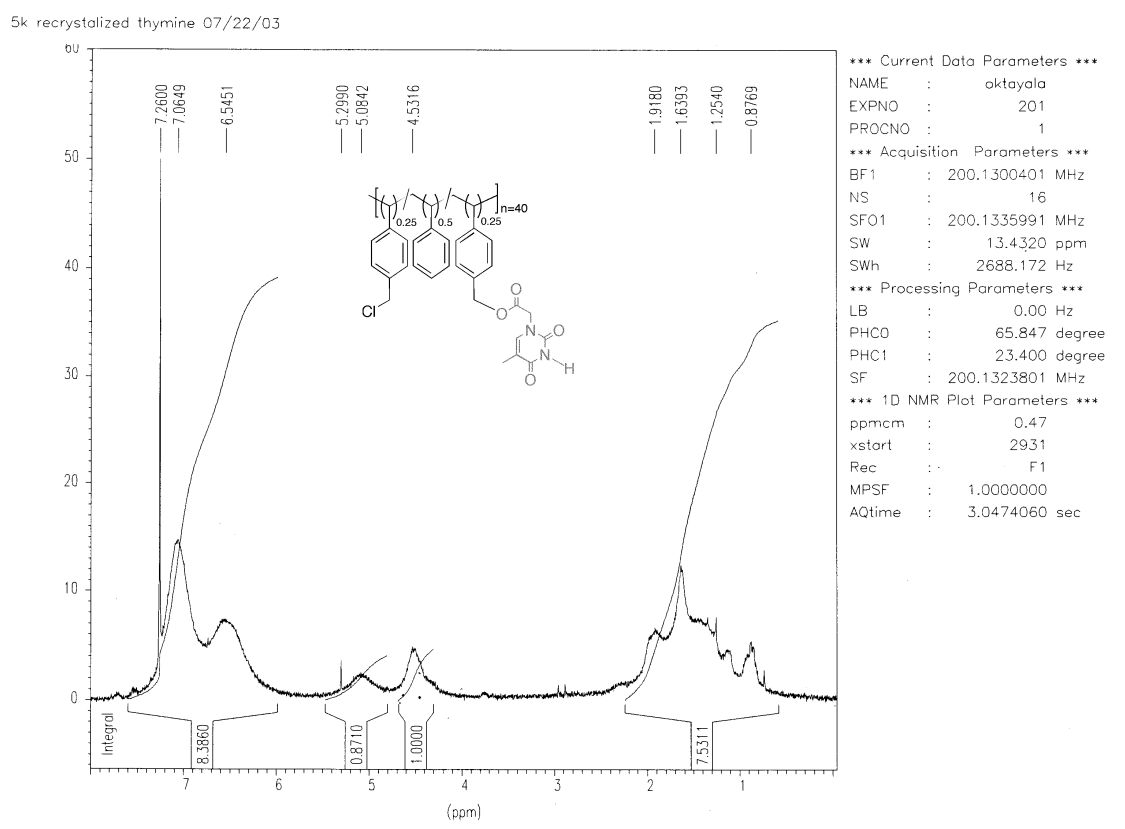

Polymer 2b PS/NMe-THY(5k,50\%)-NMR

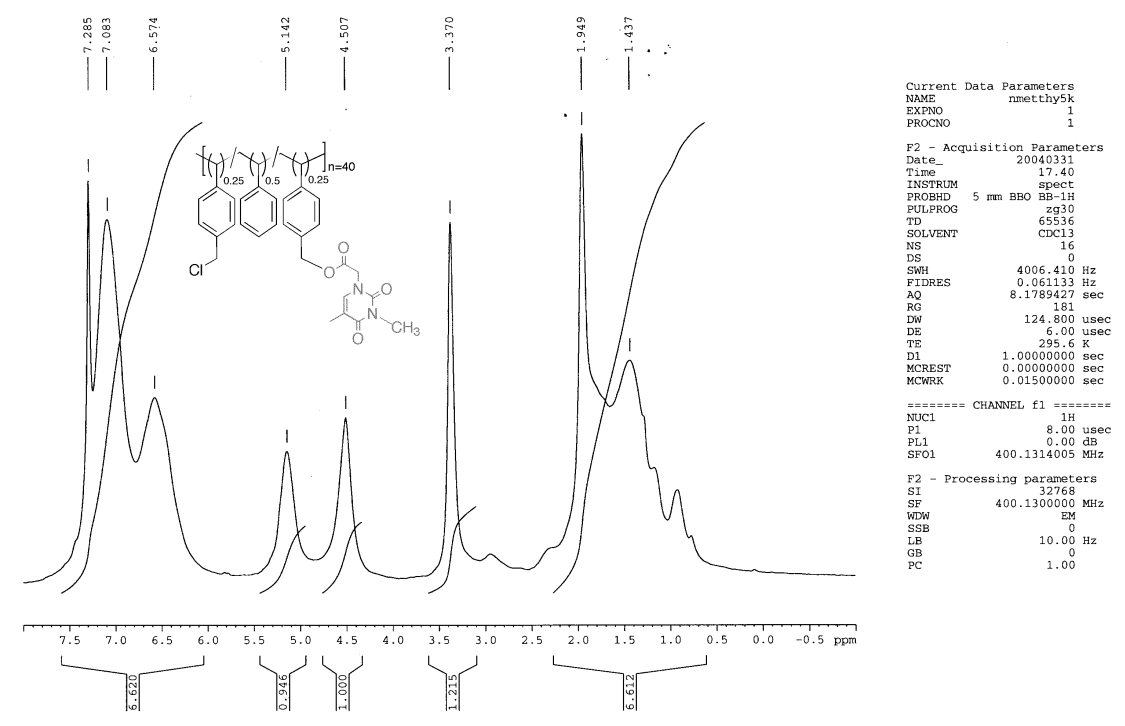


Polymer 1b chloromethylstyrene precursor PS/CMS(16k)-GPC trace

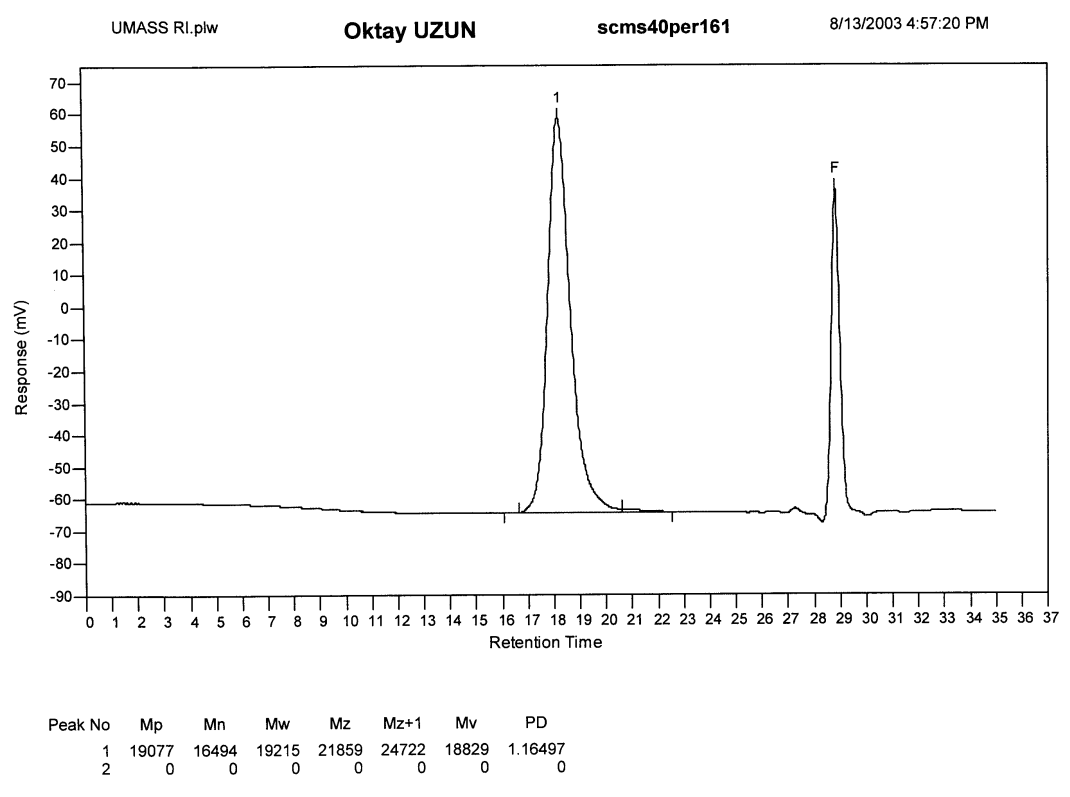

Polymer 1b chloromethylstyrene precursor PS/CMS(16k)-NMR

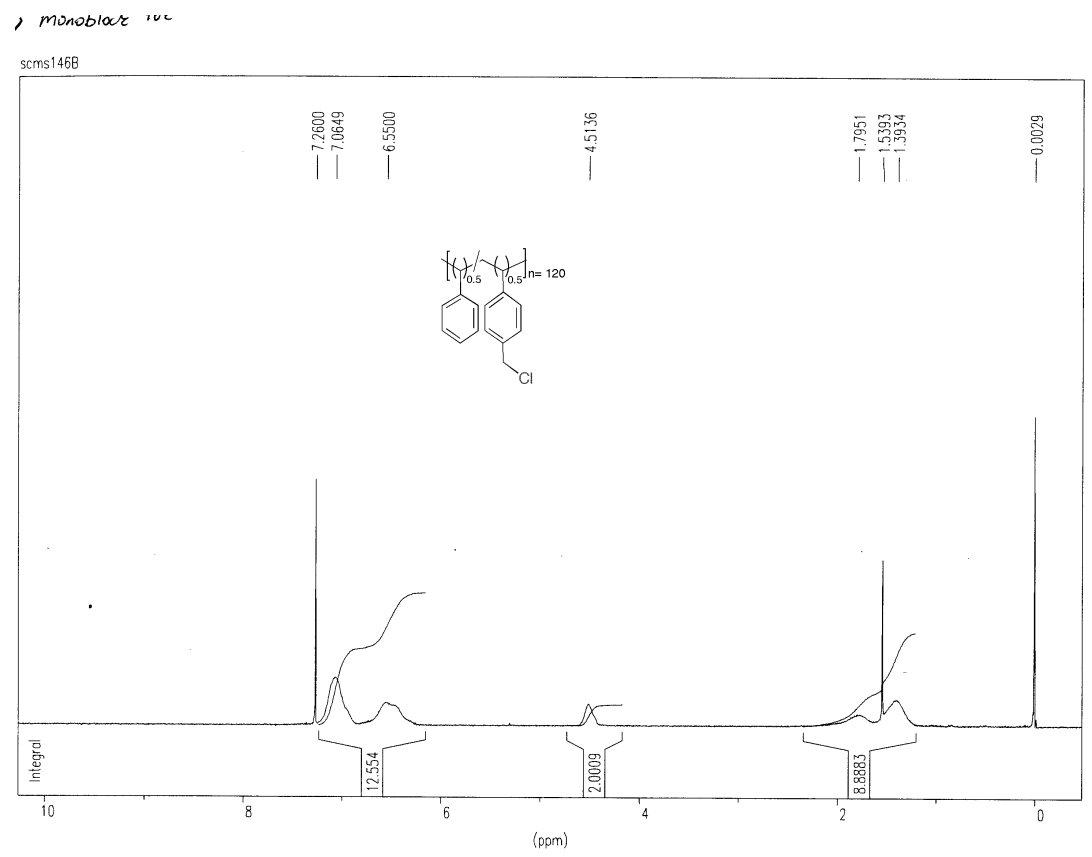


Polymer 1b PS/DAP(16k,100\%)-NMR spectra

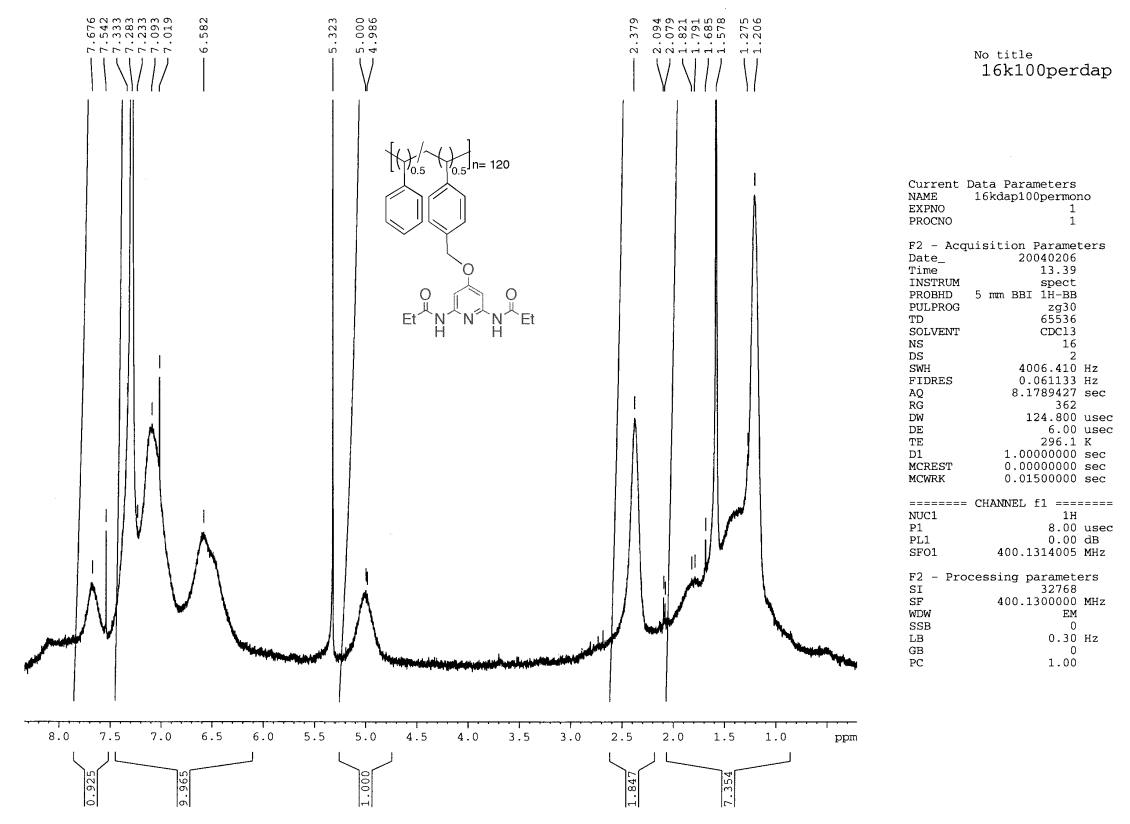

Polymer 1c chloromethylstyrene precursor PS/CMS(30k)-GPC trace

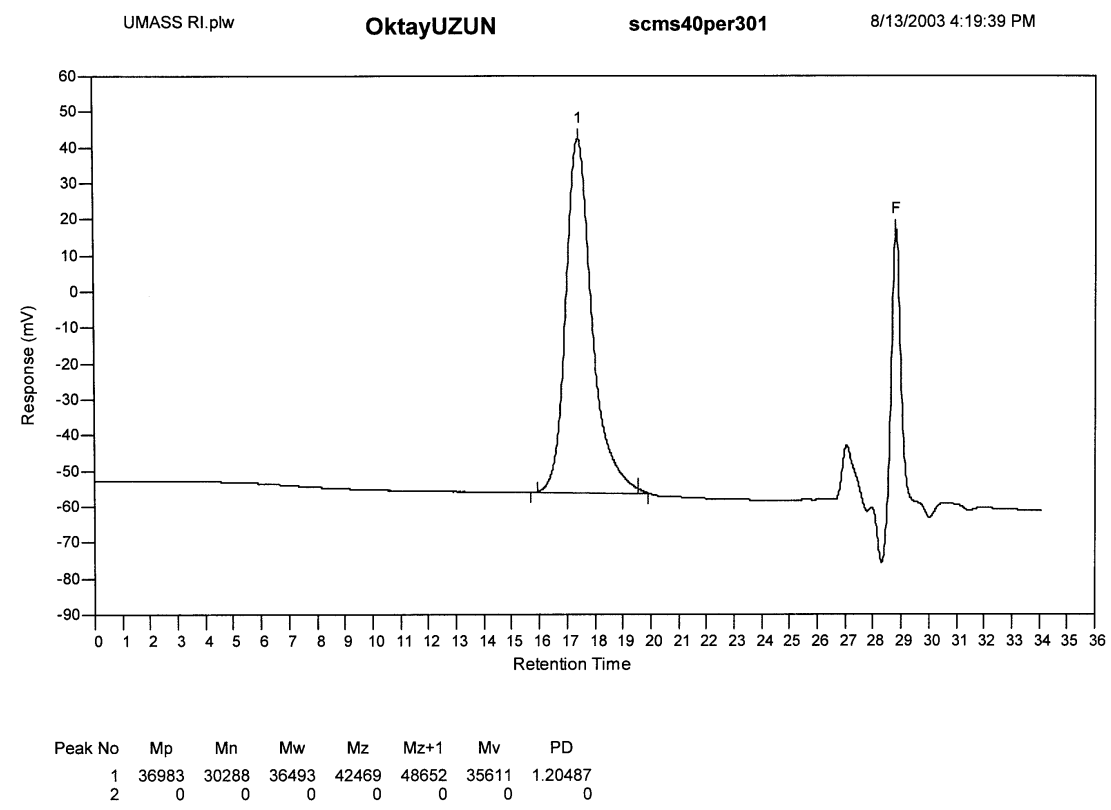


Polymer 1c chloromethylstyrene precursor PS/CMS(30k)-NMR

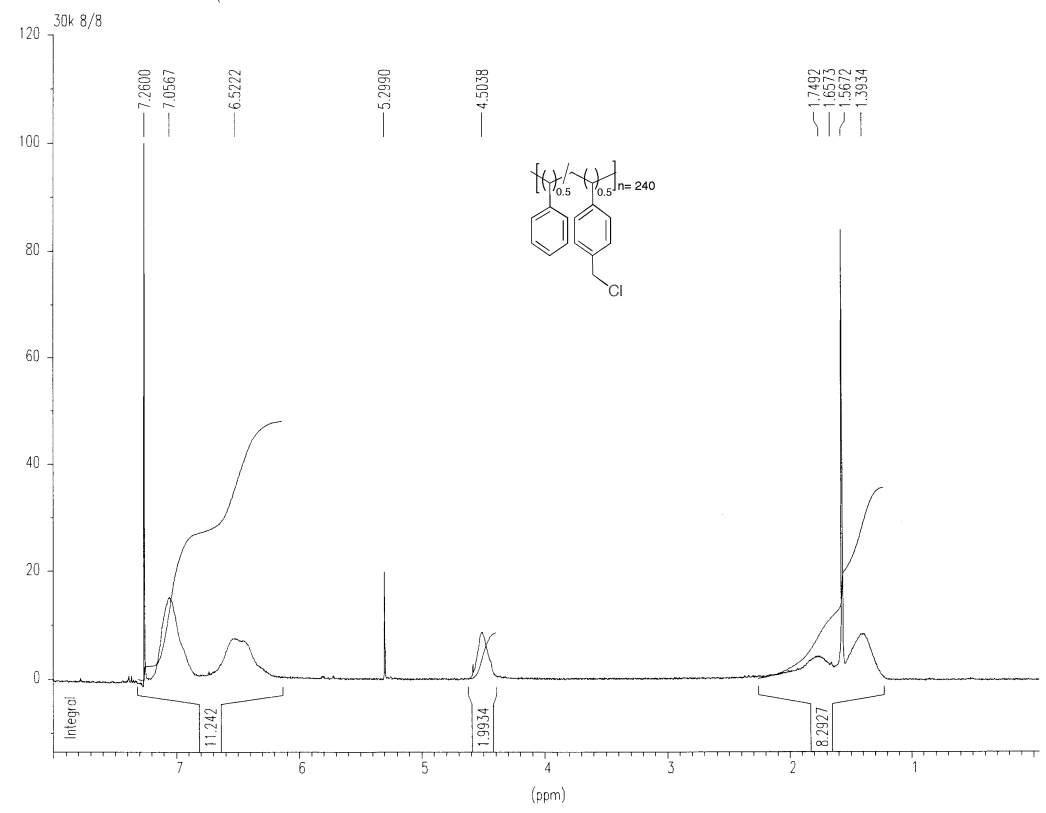

Polymer 1c PS/DAP(30k,100\%)-NMR spectra

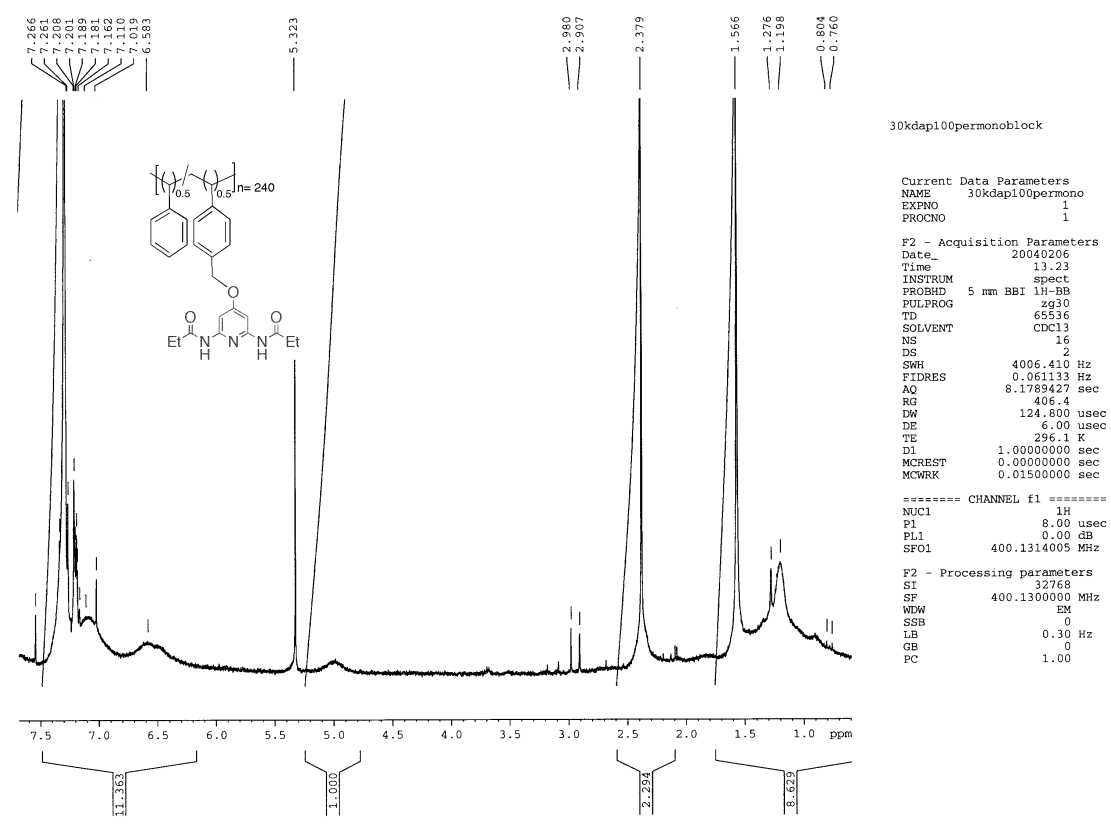




\section{IR spectra of the vesicles, polymer 1a and polymer $2 \mathrm{a}$.}

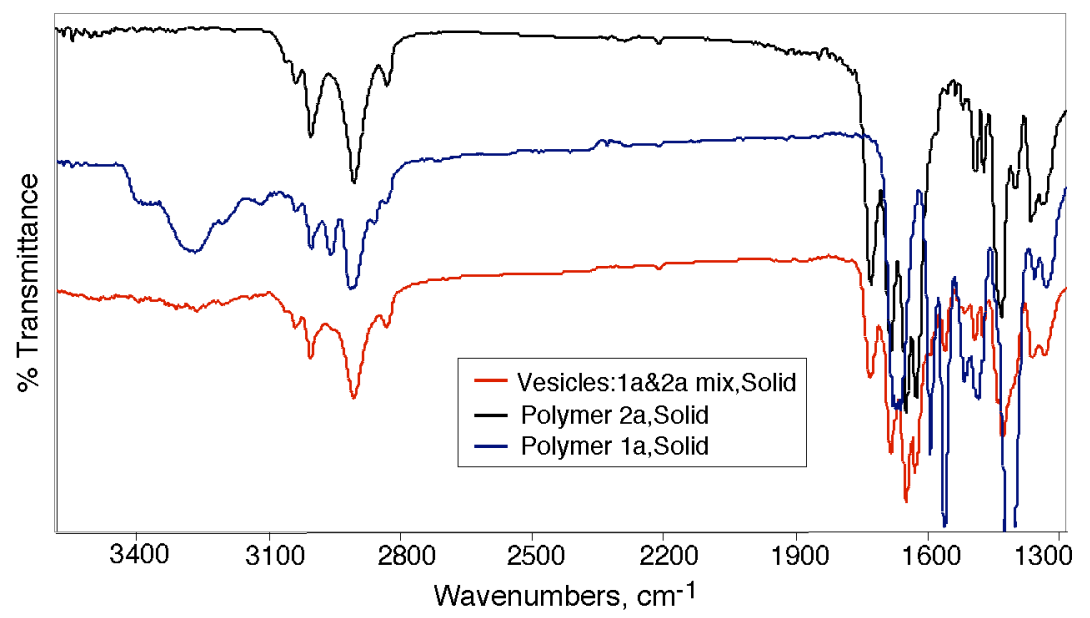

DIC Microscopy sample preparation. RIPs were prepared by mixing dropwise 3 $\mathrm{mg} / \mathrm{mL}$ solutions of Polymers $\mathbf{1}$ and $\mathbf{2}$ in $\mathrm{CHCl}_{3}$, after the addition vial was shaken for complete mixing. Transformation process took less then a second. These samples were dropped into one cavitywell microscope a 1x75x50 mm glass slide. A $22 \mathrm{~mm}$ square no. 1 cover slip was then put on the slide for observation on a Nikon E600 with dry 40x objective. Digital micrographs were taken at random to ensure a representative distribution was obtained. Images were collected over 5 minutes and the size change was not noticeable in this time scale.

LCSM sample preparation. The samples were prepared in a similar fashion as above with the following variations: a fluorescently-labeled version of polymer 1c with flavin, 2a with rhodamine B was employed. Samples were visualized on a Leica TCS SP2 Confocal Scanning Microscope.

Probability Histograms:All probability histograms were generated by fitting size distributions to the following equation. ${ }^{2}$ 


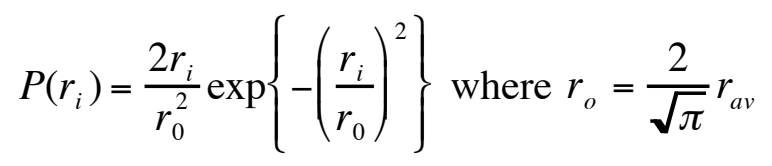

Size Distributions. Differential Interference Contrast (DIC) is used to observe translucent structures. DIC allows for acquisition of an adequate sampling population that can be converted to accurate size distributions. ${ }^{3}$ Distributions were obtained by measuring aggregates in DIC Micrographs until a suitable sampling population is obtained ( 400 aggregates).

\section{Size Distribution graphs:}

The following data are size distributions for DAP-Thy polymer combinations.

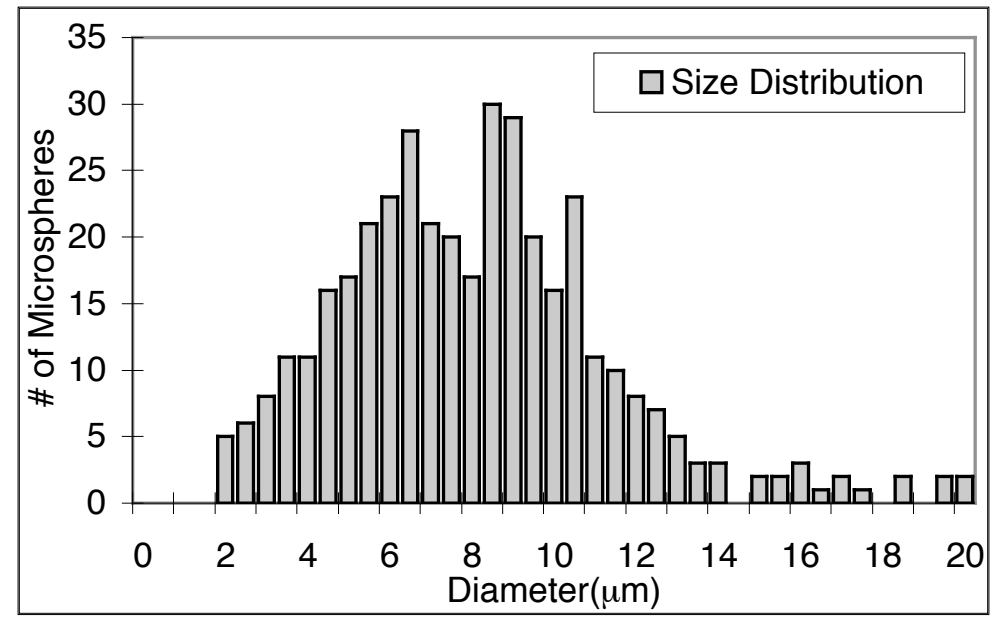

30k microspheres, reformed by adding polymer $1 \mathrm{c}$ to the preformed vesicle solution $<\mathrm{D}_{\text {microsph }}>=8.2 \pm 4.2 \square \mathrm{m}$ 


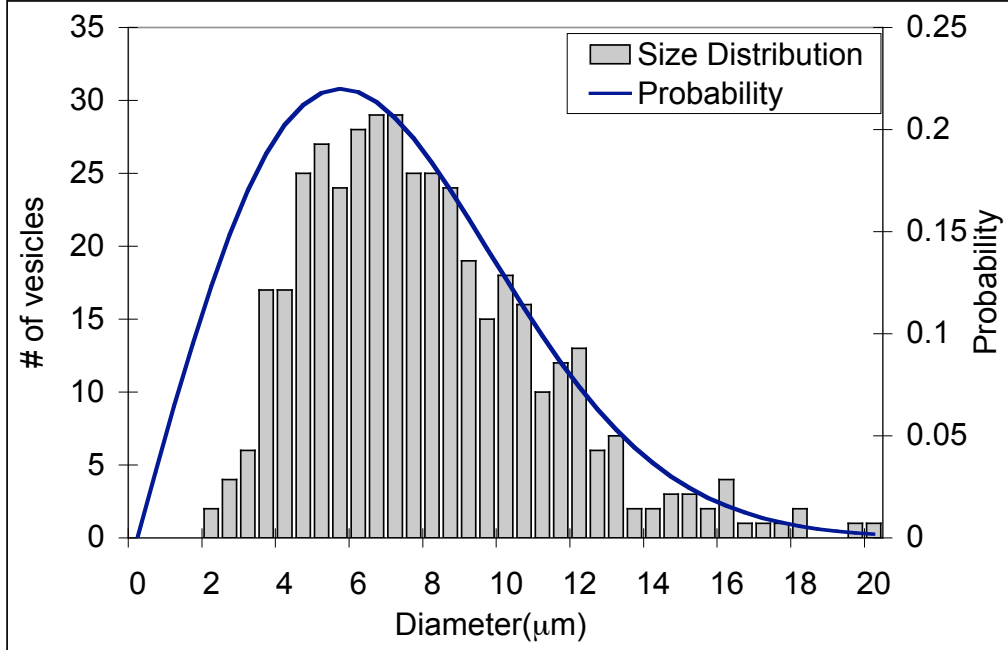

30k vesicles, reversibly formed with addition of polymer $2 \mathrm{a}$ into the microsphere solution $\left\langle\mathrm{D}_{\text {vesicle }}>=7.8 \pm 3.5 \square \mathrm{m}\right.$

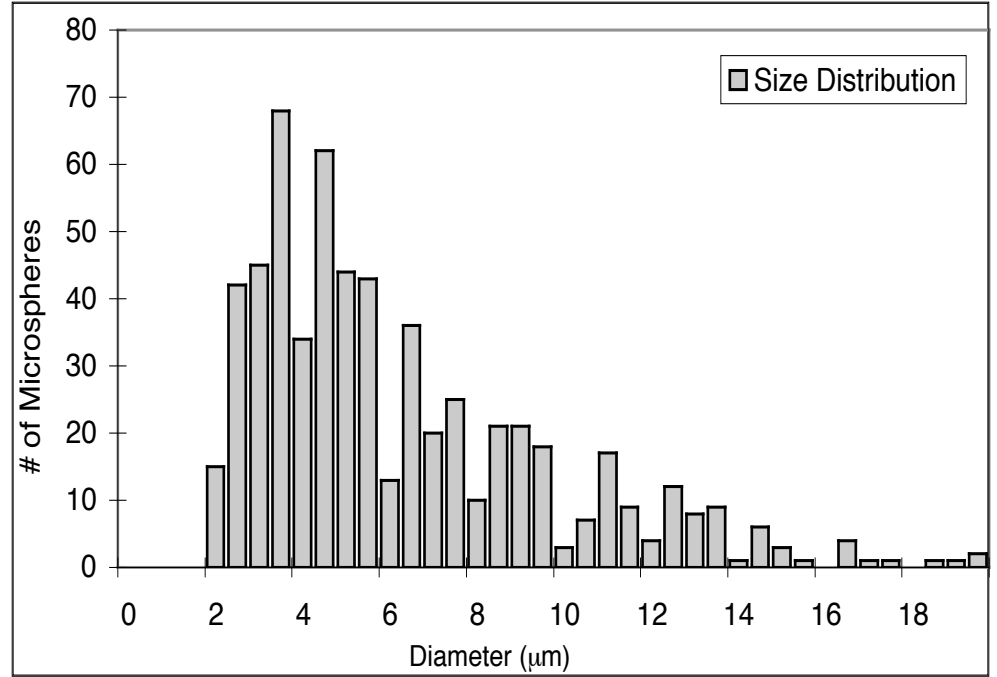

16k microspheres $<\mathrm{D}_{\text {microsph }}>=6.2 \pm 3.5 \square \mathrm{m}$ 


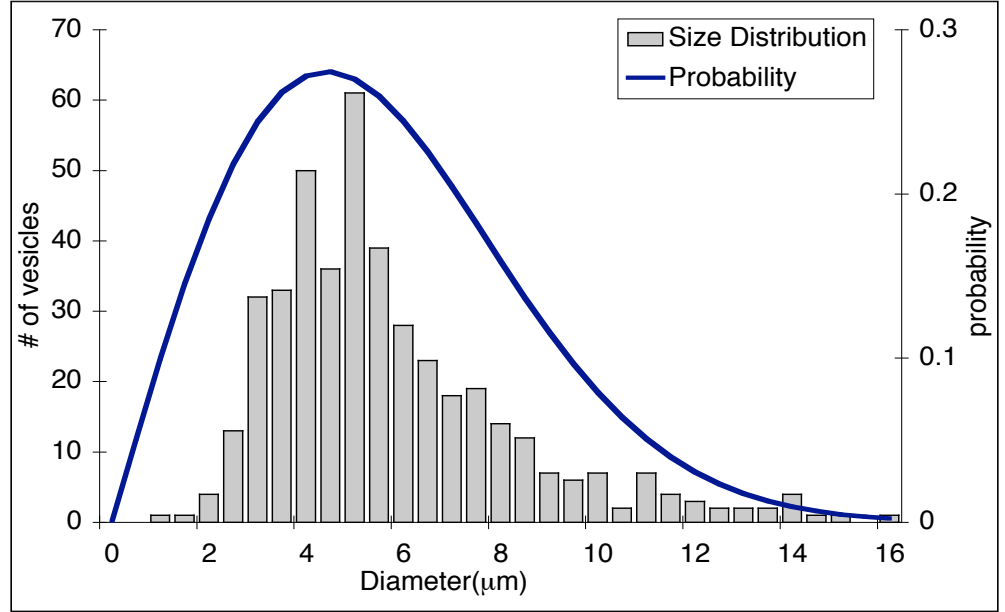

16k vesicles $\left\langle D_{\text {vesicle }}>=6.3 \pm 2.5 \square \mathrm{m}\right.$

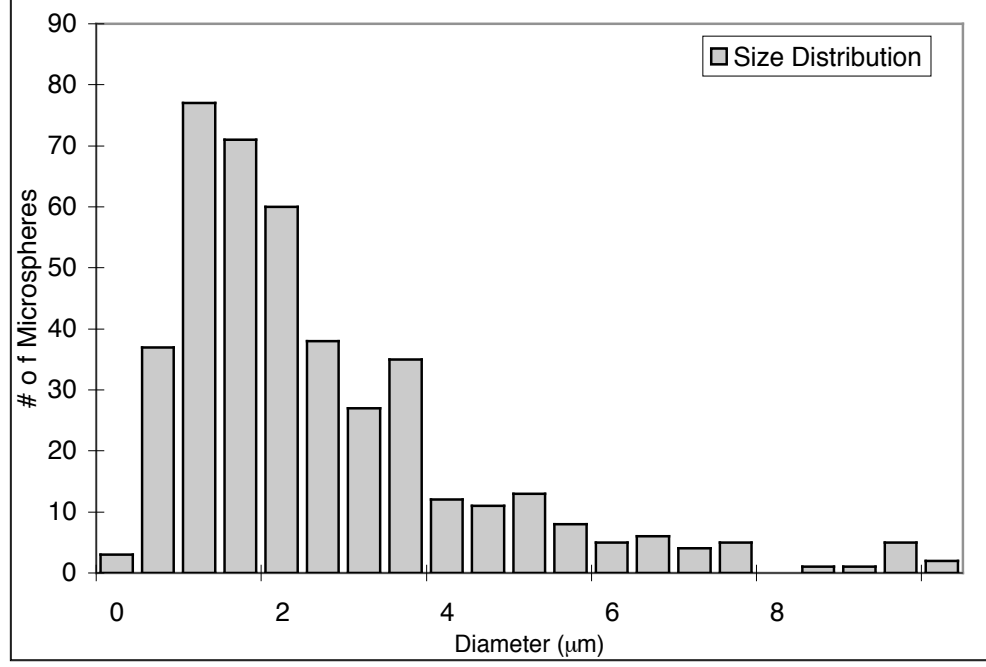

5k microspheres $<\mathrm{D}_{\text {microsph }}>=2.9 \pm 2.0 \square \mathrm{m}$ 


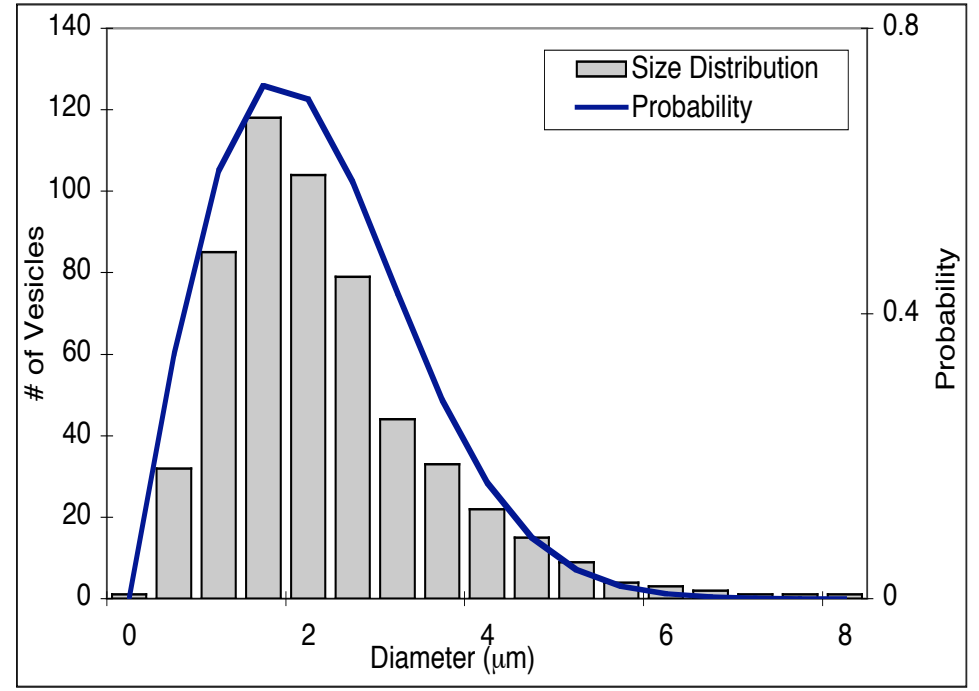

5k vesicles $<\mathrm{D}_{\text {vesicle }}>=2.4 \pm 1.1 \square \mathrm{m}$

(1) Frankamp, B. L.; Uzun, O.; Ilhan, F.; Boal, A. K.; Rotello, V. M. J. Am. Chem. Soc. 2002, 124, 892-893.

(2) Mavelli, F.; Luisi, P. L. J. Phys. Chem. 1996, 100, 16600-16607.

(3) Kachar, B.; Evans, D. F.; Ninham, B. W. J. Colloid Interface Sci. 1984, 99, 593596. 